OPEN ACCESS

Edited by:

Ming Lei,

University of Oxford, UK

Reviewed by:

Michael Franz,

Georgetown University, USA

James Alastair Fraser,

University of Cambridge, UK

*Correspondence:

Henggui Zhang,

Biological Physics Group, School of

Physics and Astronomy, The University of Manchester, Room 3.07, Schuster Building, Brunswick Street,

Manchester M13 9PL, UK

henggui.zhang@manchester.ac.uk; Ismail Adeniran,

Biological Physics Group, School of

Physics and Astronomy, The

University of Manchester, Schuster

Building, Brunswick Street,

Manchester M13 9PL, UK

ismail.adeniran@manchester.ac.uk

Specialty section: This article was submitted to Cardiac

Electrophysiology, a section of the journal Frontiers in Physiology

Received: 25 November 2014 Accepted: 26 February 2015 Published: 20 March 2015

Citation:

Adeniran I, Maclver DH, Hancox JC and Zhang $H$ (2015) Abnormal calcium homeostasis in heart failure

with preserved ejection fraction is

related to both reduced contractile function and incomplete relaxation: an electromechanically detailed biophysical modeling study. Front. Physiol. 6:78. doi: 10.3389/fphys.2015.00078

\section{Abnormal calcium homeostasis in heart failure with preserved ejection fraction is related to both reduced contractile function and incomplete relaxation: an electromechanically detailed biophysical modeling study}

\author{
Ismail Adeniran ${ }^{1 *}$, David H. Mac/ver ${ }^{1,2}$, Jules C. Hancox ${ }^{1,3}$ and Henggui Zhang ${ }^{1,4 *}$ \\ ${ }^{1}$ Biological Physics Group, School of Physics and Astronomy, The University of Manchester, Manchester, UK, ${ }^{2}$ Department \\ of Cardiology, Taunton and Somerset Hospital, Musgrove Park, Taunton, UK, ${ }^{3}$ School of Physiology and Pharmacology and \\ Cardiovascular Research Laboratories, School of Medical Sciences, Bristol, UK, ${ }^{4}$ School of Computer Science and \\ Technology, Harbin Institute of Technology, Harbin, China
}

Heart failure with preserved ejection fraction (HFpEF) accounts for about 50\% of heart failure cases. It has features of incomplete relaxation and increased stiffness of the left ventricle. Studies from clinical electrophysiology and animal experiments have found that HFpEF is associated with impaired calcium homeostasis, ion channel remodeling and concentric left ventricle hypertrophy $(\mathrm{LVH})$. However, it is still unclear how the abnormal calcium homeostasis, ion channel and structural remodeling affect the electro-mechanical dynamics of the ventricles. In this study we have developed multiscale models of the human left ventricle from single cells to the 3D organ, which take into consideration HFpEF-induced changes in calcium handling, ion channel remodeling and concentric LVH. Our simulation results suggest that at the cellular level, HFpEF reduces the systolic calcium level resulting in a reduced systolic contractile force, but elevates the diastolic calcium level resulting in an abnormal residual diastolic force. In our simulations, these abnormal electro-mechanical features of the ventricular cells became more pronounced with the increase of the heart rate. However, at the 3D organ level, the ejection fraction of the left ventricle was maintained due to the concentric LVH. The simulation results of this study mirror clinically observed features of HFpEF and provide new insights toward the understanding of the cellular bases of impaired cardiac electromechanical functions in heart failure.

Keywords: heart failure, calcium, 3D model, ventricle

\section{Introduction}

Heart failure (HF) is often categorized into two major types (Borlaug and Paulus, 2010; MacIver, 2010a; Phan et al., 2012; Liu et al., 2013; Zouein et al., 2013): HF with reduced ejection fraction (HFrEF) and HF with preserved ejection fraction (HFpEF). The main distinguishing criterion is an arbitrary cut-off value for the left ventricular ejection fraction of $>50 \%$ for HFpEF 
(Vasan and Levy, 2000; Zile et al., 2001; Yturralde and Gaasch, 2005; Asrar Ul Haq et al., 2014) and $\leq 50 \%$ for HFrEF (Borlaug and Paulus, 2010; Phan et al., 2012; Liu et al., 2013; Zouein et al., 2013). HFpEF and HFrEF are also commonly referred to as diastolic and systolic HF respectively and also share a variety of abnormalities (Borlaug and Paulus, 2010; Soma, 2011; MacIver and Dayer, 2012; Asrar Ul Haq et al., 2014). Currently, the prevalence of HFpEF is $\sim 50 \%$ (Konstantinou et al., 2013; Liu et al., 2013; Asrar Ul Haq et al., 2014), but it is predicted to become the dominant form of HF within the next decade (Liu et al., 2013; Asrar Ul Haq et al., 2014).

The leading cause of HFpEF is hypertension and the dominant pathophysiological mechanism is thought to involve impaired relaxation of the left ventricle (LV) (Liu et al., 2013; Asrar Ul Haq et al., 2014). Other distinguishing features cited are increased LV stiffness and elevated LV end-diastolic pressures (Liu et al., 2013; Asrar Ul Haq et al., 2014). In addition, abnormalities of systolic shortening are common with reduced global longitudinal strain, strain rate, reduced midwall fractional shortening, reduced systolic annular motion, increased isovolumetric contraction time and reduced systolic longitudinal shortening velocities in HFpEF (Sanderson, 2007; Wang et al., 2008; Kono et al., 2009; MacIver, 2010a). HFpEF is also characterized by dyspnoea, fluid retention, exercise intolerance, coronary artery disease and atrial fibrillation (Redfield et al., 2003; Bhatia et al., 2006; Owan et al., 2006; Konstantinou et al., 2013; Liu et al., 2013). It has a greater prevalence in older people and females (Liu et al., 2013). Patients also exhibit other comorbidities such as diabetes, obesity, peptic ulcer disease, cancer and chronic obstructive pulmonary disease (Liu et al., 2013).

As relaxation is an active energy-consuming process and dependent on intracellular $\mathrm{Ca}^{2+}$ homeostasis (Robertson et al., 1982; Ebashi, 1984; Barry and Bridge, 1993; Bers, 2001; Konstantinou et al., 2013), impaired relaxation in HFpEF may reflect abnormal intracellular $\mathrm{Ca}^{2+}$ homeostasis. Indeed, experimental data from clinical electrophysiology and animal model studies have shown that HFpEF is associated with abnormal $\mathrm{Ca}^{2+}$ handling, including an increased SR $\mathrm{Ca}^{2+}$ leak current $\left(I_{\text {leak }}\right)$ and a decreased $\mathrm{Ca}^{2+}$ release from ryanodine receptors (Borbély et al., 2005; Selby et al., 2011; Zile and Gaasch, 2011; Trenor et al., 2012; Gomez et al., 2014). In addition, some ionic currents responsible for generating cardiac action potentials are also remodeled, including those carried by the late-sodium channel, the transient outward $\mathrm{K}^{+}$channel, the inward rectifier $\mathrm{K}^{+}$channel, the $\mathrm{Na}^{+} / \mathrm{K}^{+}$pump $\left(I_{\mathrm{NaK}}\right)$, the background $\mathrm{Ca}^{2+}$ channel and the $\mathrm{Na}^{+} / \mathrm{Ca}^{2+}$ exchanger (Borbély et al., 2005; Selby et al., 2011; Zile and Gaasch, 2011; Gomez et al., 2014; Trenor et al., 2012). Structurally, HFpEF is also associated with concentric LV hypertrophy with high LV mass/volume ratio, cardiomyocyte hypertrophy and interstitial fibrosis. This is different to HFrEF, where structural remodeling is characterized by progressive ventricular dilation, eccentric LV remodeling, low LV mass/volume ratio, cardiomyocyte loss along with replacement fibrosis (Borlaug and Paulus, 2010; Konstantinou et al., 2013; Asrar Ul Haq et al., 2014).

The functional impacts of HFpEF-related abnormal $\mathrm{Ca}^{2+}$ homeostasis, ion channel and structural remodeling on cardiac electro-mechanics are unclear. Thus, the present study was conducted in order: (1) to develop a novel HFpEF electromechanical single cell model based on extant experimental data; (2) to investigate the cellular mechanisms influencing myocardial calcium homeostasis in HFpEF using an electromechanical single cell model; and (3) to evaluate the functional impacts of impaired $\mathrm{Ca}^{2+}$ handling, ion channel remodeling and degrees of concentric LV hypertrophy on the electro-mechanical activity of the heart.

\section{Materials and Methods}

\section{Electromechanical Single Cell Model}

For electrophysiology (EP), we utilized the O'Hara-Rudy (ORd) human ventricular single cell model (O'Hara et al., 2011), which was developed from undiseased human ventricular data and recapitulates human ventricular cell electrical and membrane channel properties, as well as the transmural heterogeneity of ventricular action potential (AP) across the ventricular wall (O'Hara et al., 2011). The ORd model also reproduces $\mathrm{Ca}^{2+}$ vs. voltage-dependent inactivation of L-type $\mathrm{Ca}^{2+}$ current and $\mathrm{Ca}^{2+} /$ calmodulin-dependent protein kinase II (CaMK) modulated rate dependence of $\mathrm{Ca}^{2+}$ cycling. For simulating cellular mechanics properties, we used the Tran et al. myofilament (MM) model (Tran et al., 2010). This model was chosen as it is an extension of the well-established cross-bridge cycling model of cardiac muscle contraction model of Rice et al. (2008). In addition to its ability to replicate a wide range of experimental data including steady-state force-sarcomere length (F$\mathrm{SL}$ ), force-calcium and sarcomere length-calcium relationships (Rice et al., 2008; Tran et al., 2010), it also reproduces many of the observed effects of MgATP, MgADP, $\mathrm{Pi}$, and $\mathrm{H}^{+}$on force development.

The intracellular calcium concentration from the EP model was used as the coupling link to the MM model. $\left[\mathrm{Ca}^{2+}\right]_{i}$ produced as dynamic output from the EP model during the AP served as input to the MM model from which the amount of $\mathrm{Ca}^{2+}$ bound to troponin is calculated. The formulation of the myoplasmic $\mathrm{Ca}^{2+}$ concentration in the human ventricular myocyte electromechanical cell model is:

$$
\begin{aligned}
\frac{d\left[\mathrm{Ca}^{2+}\right]_{i}}{d t}= & \beta_{C a i} \cdot\left(-\left(I_{p C a}+I_{C a b}-2 \cdot I_{N a C a, i}\right) \cdot \frac{A_{c a p}}{2 \cdot F \cdot v_{m y o}}\right. \\
& \left.-J_{u p} \cdot \frac{v_{n s r}}{v_{m y o}}+J_{\text {diff }, \mathrm{Ca}} \cdot \frac{v_{s s}}{v_{m y o}}-\frac{J_{T r o p}}{1000}\right)
\end{aligned}
$$

where $\beta_{C a i}$ is the buffer factor for $\left[C a^{2+}\right]_{i}, I_{p C a}(\mu \mathrm{A} / \mu \mathrm{F})$ is the sarcolemmal $\mathrm{Ca}^{2+}$ pump current, $I_{\mathrm{Cab}}(\mu \mathrm{A} / \mu \mathrm{F})$ is the $\mathrm{Ca}^{2+}$ background current, $I_{\mathrm{NaCa}, i}(\mu \mathrm{A} / \mu \mathrm{F})$ is the myoplasmic component of $\mathrm{Na}^{+} / \mathrm{Ca}^{2+}$ exchange current, $A_{\text {cap }}\left(\mathrm{cm}^{2}\right)$ is capacitive area, $F$ (coul $/ \mathrm{mol}$ ) is the Faraday constant, $v_{m y o}(\mu \mathrm{L})$ is the volume of the myoplasmic compartment, $v_{n s r}(\mu \mathrm{L})$ is the volume of the network sarcoplasmic reticulum compartment, $v_{s s}(\mu \mathrm{L})$ is the volume of the subspace compartment, $J_{u p}(\mathrm{mM} / \mathrm{ms})$ is the total $\mathrm{Ca}^{2+}$ uptake flux, via SERCA pump from myoplasm to the network sarcoplasmic reticulum, $J_{\text {diff }, \mathrm{Ca}}(\mathrm{mM} / \mathrm{ms})$ is the flux of the diffusion of $\mathrm{Ca}^{2+}$ from the subspace to the myoplasm and $J_{\text {Trop }}(\mu \mathrm{M} / \mathrm{ms})$ 
is the flux of $\mathrm{Ca}^{2+}$ binding to troponin calculated via the $\mathrm{MM}$ model. $\beta_{C a i}$ is formulated as:

$$
\beta_{C a i}=\frac{1}{1+\frac{[C M D N] \cdot K_{m, C M D N}}{\left(K_{m, C M D N}+\left[\mathrm{Ca}^{2+}\right]_{i}\right)^{2}}}
$$

where [CMDN] is the calmodulin $\mathrm{Ca}^{2+}$ buffer in the myoplasm and $K_{m, C M D N}$ is the half-saturation concentration of calmodulin.

\section{HFpEF Electromechanical Single Cell Model}

To develop the human electromechanical single cell model of a HFpEF cardiomyocyte, we first modified parameters of the ORd model based on HF experimental data following the work of Gomez et al. (2014), Trenor et al. (2012) on human HF. This resulted in a generic human HF model. We then made alterations based on the cellular and molecular properties of HFpEF (Zile and Gaasch, 2011) to obtain a biophysically detailed model of HFpEF. For the myofilament changes relative to control, we made modifications based on experimental data on HFpEF patients following the work of Borbély et al. (2005), Selby et al. (2011). Table 1 summarizes the extensive modifications to the control ORd cardiomyocyte to produce the HFpEF cardiomyocyte model.

\section{Parameter Sensitivity Analysis for Influential Cellular Processes on Relaxation in HFpEF}

To our knowledge, only the work by Zile and Gaasch (2011) provides any data on the differences in the cellular and molecular

TABLE 1 | Changes in original ORd model to simulate HFpEF and HFrEF.

\begin{tabular}{lcc}
\hline $\begin{array}{l}\text { Parameter } \\
\text { modified }\end{array}$ & $\begin{array}{c}\text { \% change in the HFrEF } \\
\text { model compared to } \\
\text { the normal model }\end{array}$ & $\begin{array}{c}\% \text { change in the HFpEF } \\
\text { model compared to } \\
\text { the normal model }\end{array}$ \\
\hline$I_{\text {NaL }}$ & $180 \%$ & $180 \%$ \\
$\tau_{\mathrm{hL}}$ & $180 \%$ & $180 \%$ \\
$I_{\text {to }}$ & $40 \%$ & $40 \%$ \\
$I_{\mathrm{K} 1}$ & $68 \%$ & $68 \%$ \\
$I_{\text {NaK }}$ & $70 \%$ & $70 \%$ \\
$I_{\text {Nab }}$ & $100 \%$ & $100 \%$ \\
$I_{\text {NCX }}$ & $175 \%$ & $70 \%$ \\
$I_{\text {leak }}$ & $130 \%$ & $130 \%$ \\
CaMKa & $150 \%$ & $150 \%$ \\
$J_{\text {rel, NP, } \infty}$ & $80 \%$ & $80 \%$
\end{tabular}

Myofilament (Borbély et al., 2005; Zile and Gaasch, 2011)

\begin{tabular}{lll}
\hline PCon_titin & $\times 1.00$ & $\times 2.00$ \\
PCon_collagen & $\times 2.36$ & $\times 2.00$ \\
P_Exp_collagen & $\times 0.42$ & $\times 0.50$ \\
\hline
\end{tabular}

The modified parameters are: the late $\mathrm{Na}^{+}$current $\left(I_{\mathrm{NaL}}\right)$, the time constant of inactivation of the $I_{\mathrm{NaL}}\left(t_{\mathrm{hL}}\right)$, the transient outward $K^{+}$current $\left(I_{\text {to }}\right)$, the inward rectifier $K^{+}$current $\left(I_{K 1}\right)$, the $\mathrm{Na}^{+} / \mathrm{K}^{+}$pump current $\left(I_{\mathrm{NaK}}\right)$, the background $\mathrm{Ca}^{2+}$ current $\left(I_{\mathrm{Cab}}\right)$, the $\mathrm{Na}^{+} / \mathrm{Ca}^{2+}$ exchanger ( $\left.I_{N C X}\right)$, the SR Ca ${ }^{2+}$ leak current (l leak $)$, the $\mathrm{Ca}^{2+} /$ calmodulin-dependent protein kinase II (CaMK), the nonphosphorylated $\mathrm{Ca}^{2+}$ release, via ryanodine receptors $\left(\mathrm{J}_{\text {rel, }} \mathrm{NP}, \infty\right)$, passive force factor due to titin (PCon_titin), passive force (F_passive) and active force (F_active). Based on Borbély et al. (2005), Zile and Gaasch (2011), Trenor et al. (2012) and Gomez et al. (2014). processes that influence $\mathrm{Ca}^{2+}$ homeostasis in patients with systolic and diastolic HF. We based our HFpEF model on their work (Zile and Gaasch, 2011) as given in section HFpEF Electromechanical Single Cell Model. In simulations, the only difference between the HFrEF and HFpEF models is a reduction in NCX in the HFpEF condition (see Table 1) as compared to the HFrEF condition. In order to determine whether a reduction in NCX is responsible for the poor end-diastolic relaxation in HFpEF, we performed a parameter-dependent sensitivity analysis of the HFpEF model to NCX, by varying NCX from 70\% (HFpEF) to $175 \%$ (HFrEF).

\section{Protocols}

The pacing protocols used to evaluate $\mathrm{Ca}^{2+}$ homeostasis were as follows:

\section{Post-Rest Contractions}

We used a post-rest contraction (PRC) protocol to evaluate sarcoplasmic reticulum (SR) $\mathrm{Ca}^{2+}$ content, retention, release, reuptake, and leak. After pacing the single cell for $10 \mathrm{~min}$ at $1 \mathrm{~Hz}$ to allow steady-conditions to be attained, resting intervals of $1,2,3$, 5 , and $10 \mathrm{~s}$ were introduced. The resting periods were then followed by a single stimulus. The varying developed indices such as active tension are a reflection of $\mathrm{SR} \mathrm{Ca}^{2+}$ release.

\section{Tissue Mechanics Model}

We modeled cardiac tissue mechanics within the theoretical framework of nonlinear elasticity (Marsden and Hughes, 1994; Holzapfel, 2000) as an inhomogeneous, anisotropic, nearly incompressible nonlinear material similar to previous studies (Costa et al., 2001; Whiteley et al., 2007; Niederer and Smith, 2008; Pathmanathan and Whiteley, 2009; Adeniran et al., 2013a,b). We used a two-field variational principle with the deformation $u$ and the hydrostatic pressure $p$ as the two fields (Bonet and Wood, 2008; Adeniran et al., 2013a,b; Le Tallec). $p$ is utilized as the Lagrange multiplier to enforce the near incompressibility constraint. Thus, the total potential energy function $\Pi$ for the mechanics problem is formulated as:

$$
\Pi(u, p)=\Pi_{\text {int }}(u, p)+\Pi_{\text {ext }}(u)
$$

where $\Pi_{\text {int }}(u, p)$ is the internal potential energy or total strain energy of the body and $\Pi_{e x t}(u)$ is the external potential energy or potential energy of the external loading of the body. As in previous studies (Niederer and Smith, 2008; Keldermann et al., 2009; Pathmanathan and Whiteley, 2009; Adeniran et al., 2013a,b), in the absence of body forces, and assuming that the body is always in instantaneous equilibrium and no inertia effects, the coordinates of the deformed body satisfies the steady-state equilibrium equation with near incompressibility enforced.

The values that minimize the total potential energy in Equation (3) are obtained by searching for its critical points in suitable admissible displacement and pressure spaces $\hat{U}$ and $\hat{P}$. The corresponding Euler-Lagrange equations resulting from Equation (3) lead to solving the problem (Braess and Ming, 2005; Auricchio et al., 2010; Boffi et al., 2013; Le Tallec): 
Find $(u, p)$ in $\hat{U} \times \hat{P}$ such that:

$$
\begin{array}{r}
\int_{\Omega} \frac{\partial \hat{W}}{d F}(x, I d+\nabla u): \nabla v d x-\int_{\Omega} p \frac{\partial \operatorname{det}}{d F}(x, I d+\nabla u): \nabla v d x \\
=\int_{\partial \Omega} g \cdot v d s \quad \forall v, u \in \hat{U} \\
\int_{\Omega} q[\operatorname{det}(I d+\nabla u)-1] d x=0 \quad \forall v, u \in \hat{P}
\end{array}
$$

where $\hat{U}$ and $\hat{P}$ are the admissible variation spaces for the displacements and the pressures, respectively. $F=I d+\nabla u$ is the deformation gradient, $v$ is a test function and $\hat{W}$ is the material stored energy function and corresponds to the density of elastic energy locally stored in the body during the deformation.

The deformation gradient $F$ is a tensor that maps elements from the undeformed configuration to the deformed configuration (Marsden and Hughes, 1994; Holzapfel, 2000). Following Cherubini et al. (2008), Ambrosi et al. (2011), we multiplicatively decompose $F$ into a microscopic (active) component and a macroscopic elastic (passive) component:

$$
F=F_{e} F_{0}
$$

The active component $F_{o}$ measures the length change of the tissue due to muscle contraction while the passive component $F_{e}$ accounts for the passive mechanical response of the tissue and possible tension due to external loads.

With the vector fields $f, s$, and $n$ denoting the unique direction of the fibers, sheets and sheet-normals in the undeformed state of the LV, the microscopic active component of the deformation tensor $F$ takes the form:

$$
F_{o}=I+\gamma_{f} f \otimes f+\gamma_{s} s \otimes s+\gamma n \otimes n
$$

where $I$ is the identity tensor and $\gamma_{x}$ is a scalar field that represents the intensity of the contraction, i.e., the active strain in the appropriate direction. $\gamma_{f}$ is defined as:

$$
\gamma_{f}=\frac{S L-S L_{0}}{S L_{0}}
$$

where $S L$ is the new sarcomeric length and $S L_{0}$ is the resting sarcomere length of the electromechanical single cell. Following the work of (Rossi et al., 2014)

$$
\gamma_{n}=K \gamma_{f}, \gamma_{s}=\frac{1}{\left(1+\gamma_{f}\right)\left(1+\gamma_{n}\right)}-1
$$

Thus, $\gamma>0$ denotes elongation, and $\gamma<0$ denotes contraction. The parameter $K$ is the link between the microscopic and the macroscopic active deformations (Bogaert and Rademakers, 2001; Rossi et al., 2014) and in our simulations, we used $K=4$, according to experimental observations (Rademakers et al., 1994; Rossi et al., 2014).

The elastic component $F_{e}$ is formulated as:

$$
F_{e}=F F_{0}^{-1}
$$

and the corresponding Right Cauchy-Green strain tensor is:

$$
C_{e}=F_{e}^{T} F_{e}
$$

The associated Green-Lagrange strain tensor is:

$$
E_{e}=\frac{1}{2}\left(C_{e}-I\right)
$$

To characterize the constitutive behavior of cardiac tissue, we used a mixed formulation of the compressible neo-Hookean strain energy function $W$ (Auricchio et al., 2010):

$$
\hat{W}=\frac{\mu}{2}[I: \hat{C}-d]-\mu \ln \hat{J}+p \ln \hat{J}-\frac{p^{2}}{2 \lambda}
$$

where $\mu$ and $\lambda$ are positive constants, “:” represents the usual inner product for second-order tensors, $\hat{C}$ is the right CauchyGreen deformation tensor, $d$ is the spatial dimension and $\hat{J}=$ $\operatorname{det} \hat{F}$ is the Jacobian of the deformation gradient.

\section{Tissue Electrophysiology Model}

The monodomain representation (Colli Franzone et al., 2005; Keener and Sneyd, 2008; Adeniran et al., 2013a,b) of cardiac tissue was used for the electrophysiology model:

$$
C_{m} \frac{d v}{d t}=-\left(I_{\text {ion }}+I_{\text {stim }}\right)+\nabla \cdot(D \nabla V)
$$

where $C_{m}$ is the cell capacitance per unit surface area, $V$ is the membrane potential, $I_{\text {ion }}$ is the sum of all transmembrane ionic currents from the electromechanics single cell model, $I_{\text {stim }}$ is an externally applied stimulus and $D$ is the anisotropic diffusion tensor given by:

$$
D=\sigma_{f}(f \otimes f)+\sigma_{s}(s \otimes s)+\sigma_{n}(n \otimes n)
$$

$\sigma_{f}, \sigma_{f}$, and $\sigma_{f}$ are the intracellular conductivities in the fiber, sheet and cross-sheet directions respectively. These were set to $3.0,0.1$, and $0.31525 \mathrm{~ms} \cdot \mathrm{mm}^{-1}$ respectively. These gave a conduction velocity of $65 \mathrm{~cm} \cdot \mathrm{s}^{-1}$ in the fiber direction along multiple cells, which is close to the value $70 \mathrm{~cm} \cdot \mathrm{s}^{-1}$ observed in the fiber direction in human myocardium (Taggart et al., 2000). To guard against any drift in the steady state values of the ion concentrations in the model, the electromechanical single cell models described in sections Electromechanical Single Cell Model to HFpEF Electromechanical Single Cell Model were pre-paced for a 1000 beats before being incorporated into the tissue model.

\section{Geometry and Meshes}

For unbiased comparison between increasing severity of concentric LV hypertrophy in HFpEF, we used truncated ellipsoids for the 3D simulations (Figure 1A). Each LV geometry was segmented into distinct endocardial (60\%), mid-myocardial (30\%), and epicardial (10\%) regions. The chosen cell proportion in each region reflects experimental data for cells spanning the left ventricular wall of the human heart (Drouin et al., 1995). There exists some controversy on the existence and functional role of 


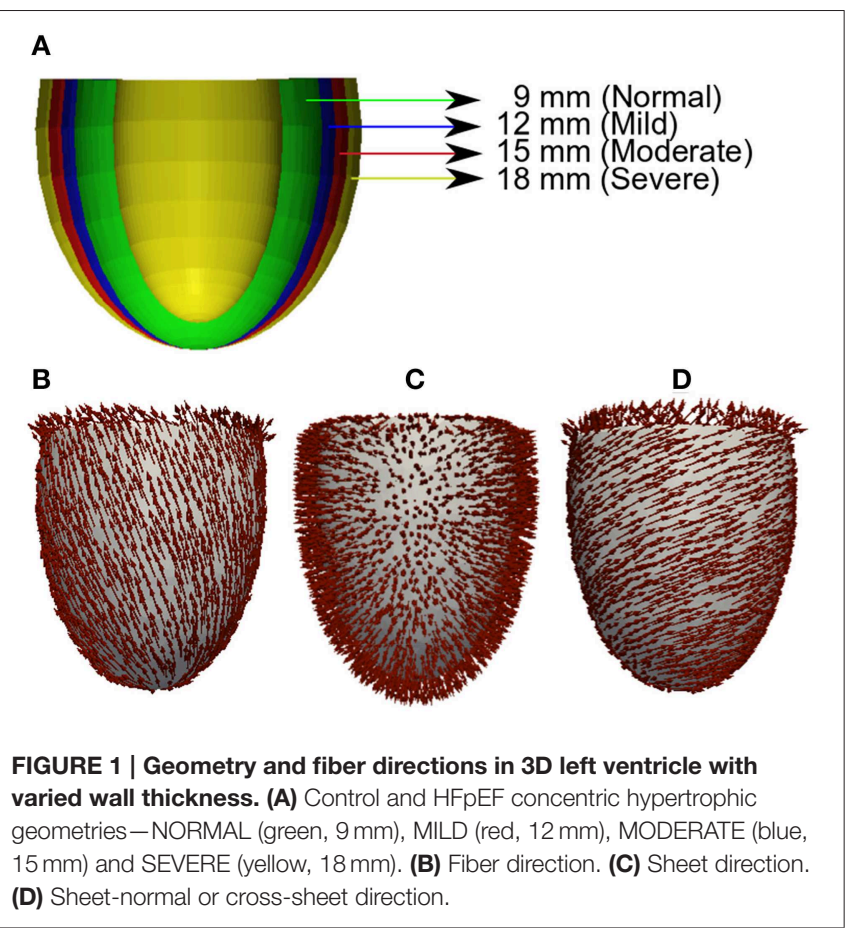

MCELLs in the human heart (Wilson et al., 2011). However, we included MCELLs in our model on the basis of studies that give evidence of their existence in cells isolated from the right ventricle of patients with heart failure (Li et al., 1998) and in a perfused piece of left ventricular wall ("wedge preparation") (Drouin et al., 1995). The conditional activation sites were projected from those of a healthy 34-year old male determined empirically across the ventricle wall and were validated by reproducing the activation sequence and QRS complex in the measured 64-channel ECG (Keller et al., 2009) of that person. Figures 1B-D shows the anisotropic fiber fields reconstructed by the algorithm in Rossi et al. (2014) for the fiber (Figure 1B), sheet (Figure 1C), and cross-sheet (Figure 1D) directions.

\section{Solving the Electromechanics Problem}

The electromechanics problem consists of two sub-problems: the electrophysiology problem and the mechanics problem. The electrophysiology problem (Equation 14) was solved with a Strang splitting method (Sundnes et al., 2005) ensuring that the solution is second-order accurate. It was discretized in time using the Crank-Nicholson method (Burnett, 1987), which is also secondorder accurate and discretized in space with Finite Elements (Ciarlet, 2002; Braess, 2007; Brenner and Scott, 2010). $I_{\text {ion }}$ in Equation (14) represents the single cell electromechanics model from which the active strain input to the $3 \mathrm{D}$ mechanics model for contraction is obtained. The system of ordinary differential equations (ODE) composing $I_{\text {ion }}$ was solved with a combination of the Rush-Larsen scheme (Rush and Larsen, 1978) and the CVODE solver (Cohen and Hindmarsh, 1996; Alan and Hindmarsh, 2005).

The mechanics problem (Equation 2) was also solved using the Finite element Method using the automated scientific computing library, FEniCS (Logg et al., 2012). The resulting nonlinear system of equations was solved iteratively using Newton's method to determine the equilibrium configuration of the system. Over a typical finite element domain, $P_{2}$ elements (Braess, 2007; Brenner and Scott, 2010; Ern and Guermond, 2010; Boffi et al., 2013) were used to discretize the displacement variable $u$, while the pressure variable $p$ was discretized with $P_{1}$ elements (Braess, 2007; Brenner and Scott, 2010; Ern and Guermond, 2010; Boffi et al., 2013). This $P_{2}-P_{1}$ mixed finite element has been proven to ensure stability (Chamberland et al., 2010; Haga et al., 2012; Logg et al., 2012) and an optimal convergence rate (Hughes, 2000; Chamberland et al., 2010; Ern and Guermond, 2010).

The algorithm for solving the full electromechanics problem is as follows:

(1) While time < tend:

(a) Solve the electrophysiology problem for $\Delta t_{\text {mechanics }}=$ $1 \mathrm{~ms}$ with active strain $\gamma$ as output $\left(\Delta \mathrm{t}_{\text {electrophysiology }}=\right.$ $0.01 \mathrm{~ms}$ ).

(b) Project $\gamma$ from the electrophysiology mesh onto the mechanics mesh.

(2) Solve the mechanics problem with $\gamma$ as input.

\section{Results}

\section{Model Validation}

To validate the HFpEF model, we computed a steady-state forcecalcium (F-pCa) relation for a sarcomere length (SL) of $2.2 \mu \mathrm{m}$ for comparison with prior experimental data (Borbély et al., 2005). Results are shown in Figure 2. The model reproduced the differences in total and passive forces between control and HFpEF, which matched experimental data (inset).

\section{Functional Consequences of HFpEF Transmurally in the LV}

Figure 3 shows the electromechanical consequences of $\mathrm{HFpEF}$ in a free-running cell from each of the epicardium (EPI) (Figures 3Ai-Aiv), mid-myocardium (MCELL) (Figures 3Bi-Biv) and endocardium (ENDO) (Figures 3Ci-Civ). HFpEF increased the action potential duration $\left(\mathrm{APD}_{90}\right)$ in each of the EPI (Figure 3Bi), MCELL (Figure 3Bii) and ENDO (Figure 3Bi) cell types. APD 90 was increased from 233 to $262 \mathrm{~ms}$ in EPI, 357 to $439 \mathrm{~ms}$ in MCELL and 274 to $348 \mathrm{~ms}$ in ENDO. These differences in $\mathrm{APD}_{90}$ across the ventricular wall are suggestive of possible T-wave changes and altered dispersion of repolarization in HFpEF. The cytosolic $\mathrm{Ca}^{2+}$ concentration $\left[\mathrm{Ca}^{2+}\right]_{i}$ was reduced in amplitude in all the cell types (Figures 3Aii-Cii), which led to a decrease in the SL shortening (Figures 3Aiii-Ciii) and consequently, a reduction in the active force (Figures $\mathbf{3 A i v}-\mathbf{C i v}$ ). Of note is that the diastolic $\left[\mathrm{Ca}^{2+}\right]_{i}$ level was increased in HFpEF compared to control (Figures 3Aii-Cii) leading to incomplete relaxation (Figures 3Aiv-Civ). This electromechanical model predicted a combination of reduced contractile force and stress generation and incomplete relaxation as the pathophysiological mechanism of HFpEF (Zile et al., 2004; Borlaug and Paulus, 2010; Phan et al., 


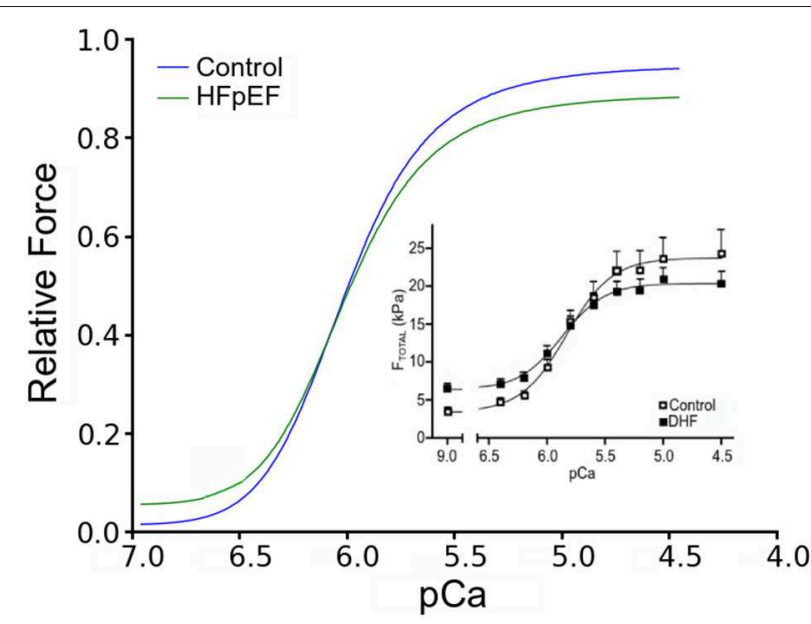

FIGURE 2 | Force-pCa relationship. Simulated Force-pCa relation of control and HFpEF. Relative force is normalized to maximum value in control. Inset: Experimental force-pCa relation from human patients adopted from Borbély et al. (2005).

2012; Konstantinou et al., 2013; Liu et al., 2013; Zouein et al., 2013; Asrar Ul Haq et al., 2014).

\section{Functional Consequences of HFpEF on Ionic Currents Compared with HFrEF}

Figure 4 shows action potentials in control, HFrEF and HFpEF conditions (Figure 4A) computed from the EPI cell model, together with the corresponding ionic currents of $I_{\mathrm{CaL}}$ (Figure 4B), $I_{\mathrm{NaCa}}$ (Figure 4C), the SR content (Figure 4D), $I_{p C a}$ (Figure 4E), intracellular $\mathrm{Na}^{+}\left(\left[\mathrm{Na}^{+}\right]_{i}\right.$; Figure $\left.4 \mathbf{F}\right)$ and $\mathrm{K}^{+}$ $\left(\left[\mathrm{K}^{+}\right]_{i}\right.$; Figure 4G) concentrations and $\mathrm{I}_{\mathrm{Na}, K}$. In comparison to the control condition, HFpEF and HFrEF increased the APD, which can be attributable to the increased inward $\mathrm{Na}^{+}$current $\left(\mathrm{I}_{\mathrm{NaL}}\right)$ and decreased outward $\mathrm{K}^{+}$current $\left(\mathrm{I}_{\mathrm{K} 1}\right.$ and $\mathrm{I}_{\mathrm{NaK}}$; see Table 1) (Figure 4H) (Glitsch, 2001; Workman et al., 2003; Bueno-Orovio et al., 2014). Accompanying these changes were increased $\left[\mathrm{Na}^{+}\right]_{i}$ (Figure 4F) and reduced $\left[\mathrm{K}^{+}\right]_{i}$ (Figure 4G). The difference in the amplitude of $I_{\mathrm{CaL}}$ among control, HFpEF and HFrEF was small, but its duration was longer in HFpEF and longest in HFrEF (Figure 4B), possibly due to a secondary effect of a prolonged APD. In the HFpEF condition, a decreased $I_{\mathrm{NaCa}}$ was observed, partially due to reduced $\mathrm{Na}^{+}-\mathrm{Ca}^{2+}$ exchanger (NCX) (see Table 1) and partially due to the reduction in the $\mathrm{Na}^{+}-\mathrm{K}^{+}$pump activity as it indirectly regulated $\mathrm{Ca}^{2+}$ extrusion by the NCX (Barry et al., 1985; Bueno-Orovio et al., 2014). This resulted in a reduced sarcoplasmic reticulum (SR) content in HFpEF (Figure 4D), and consequently leading to a smaller SR $\mathrm{Ca}^{2+}$ release as demonstrated by the reduced amplitude of the SR content (Figure 4D). The activity of the sarcolemmal Ca ${ }^{2+}$ pump was increased during the diastolic period, but reduced in the systolic period (Figure 4E). These simulation results provided a cellular basis for the abnormal $\mathrm{Ca}^{2+}$ handling in HFpEF. Results from the MCELL and ENDO cell models showed similar behavior (these are shown in the Supplementary Material).

Unlike in HFpEF, there was an increased $I_{\mathrm{NaCa}}$ (Figure 4C) due to the HFrEF model formulation involved a $175 \%$ NCX increase (Table 1). Figure 4C shows that NCX removed an excessive amount of $\mathrm{Ca}^{2+}$ from the cell in its forward mode compared to control, leading to a deficit in the $\mathrm{SR} \mathrm{Ca}^{2+}$ content (Figure 4D), a greater reduction in $\left[\mathrm{Ca}^{2+}\right]_{i}$ compared to HFpEF (Figure 4J) and consequently a lower active force (Figure 4L).

\section{Post-Rest Contraction Properties of HFpEF}

Further simulations were performed to investigate the ratedependent impact of $\mathrm{HEpEF}$ on $\mathrm{Ca}^{2+}$ handling. Results are shown in Figure 5, which shows the outcome of the PRC protocol at a pacing rate of $1 \mathrm{~Hz}$ (Figures $5 \mathbf{A i}-\mathbf{A v}$ ) and $2 \mathrm{~Hz}$ (Figures 5Bi-Bv). Diastolic $\left[\mathrm{Ca}^{2+}\right]_{i}$ level in HFpEF increased by $\sim 75 \%$ relative to control at all resting intervals (Figures 5Ai,Bi). This led to an increase in resting tension (Figures 5Aiii,Biii). Though there was a significant increase in diastolic $\left.\mathrm{Ca}^{2+}\right]_{i}$ level, the SR content was lower in HFpEF than in control (Figures 5Aii,Bii) as was peak systolic tension (Figures 5Aiv,Biv). This is counter-intuitive as with an increased diastolic concentration of $\mathrm{Ca}^{2+}$ in the cytosol, one would expect greater $\mathrm{Ca}^{2+}$ sequestration into the SR, and therefore an increased $\mathrm{Ca}^{2+}$ content leading to an increased $\mathrm{Ca}^{2+}$ release from the SR, resulting in a greater $\mathrm{Ca}^{2+}$ transient amplitude and a greater systolic tension. The reduction in the peak systolic $\mathrm{Ca}^{2+}$ level and the corresponding tension and inefficient $\mathrm{SR} \mathrm{Ca}^{2+}$ activity was due to excessive leak of $\mathrm{Ca}^{2+}$ from the $\mathrm{SR}$ (Figures $\mathbf{5} \mathbf{A v}, \mathbf{B v}$ ). These results were more pronounced at a pacing rate of $2 \mathrm{~Hz}$ (Figures $5 \mathbf{B i}-\mathbf{B v}$ ) because of the shorter duration between beats allowing less recovery time for $\mathrm{Ca}^{2+}$ cycling processes.

\section{Sensitivity of Diastolic Relaxation to NCX}

In Figure 6, effects of a systematic change of NCX in HFpEF model from $70 \%$ (HEpEF) to $175 \%$ (HFrEF) on the action potentials (Figure 6A), $I_{\mathrm{NaCa}}$ (Figure 6B), $\left[\mathrm{Ca}^{2+}\right]_{i}$ (Figure 6C) and active force (Figure 6D) are shown. Diastolic relaxation was impaired when NCX was at $70 \%$ of the control value (e.g., for HFpEF), but gradually improved with increasing NCX activity to 100 and $150 \%$ of the control value. It became normal at $175 \%$ (e.g., for HFrEF; Figure 6D) of the control value. Peak relative force during systole occurred later and with lower amplitude with increasing NCX activity.

\section{D Electromechanical Consequences of HFpEF in Hypertrophic Geometries}

Figure 7 shows the results of incorporating the cellular HFpEF electromechanical models into three-dimensional truncated ellipsoid representations of the LV. Effects of varying degrees of left ventricular hypertrophy on the ejection fraction are also shown. The LV was at rest before activation $(0 \mathrm{~ms})$. At about $200 \mathrm{~ms}$, the LV was completely activated and contraction was underway in the NORMAL, MILD, MODERATE, and SEVERE conditions of hypertrophy. By $300 \mathrm{~ms}$, repolarization had commenced and the LV in all conditions was undergoing relaxation. At $700 \mathrm{~ms}$, repolarization was completed in all the conditions; however, relaxation was still on going in the hypertrophic cases but was complete in the NORMAL condition. The LVEF in NORMAL, MILD, MODERATE, and SEVERE was 58, 57, 63, and 71\% respectively showing that LVEF was increased with increasing 

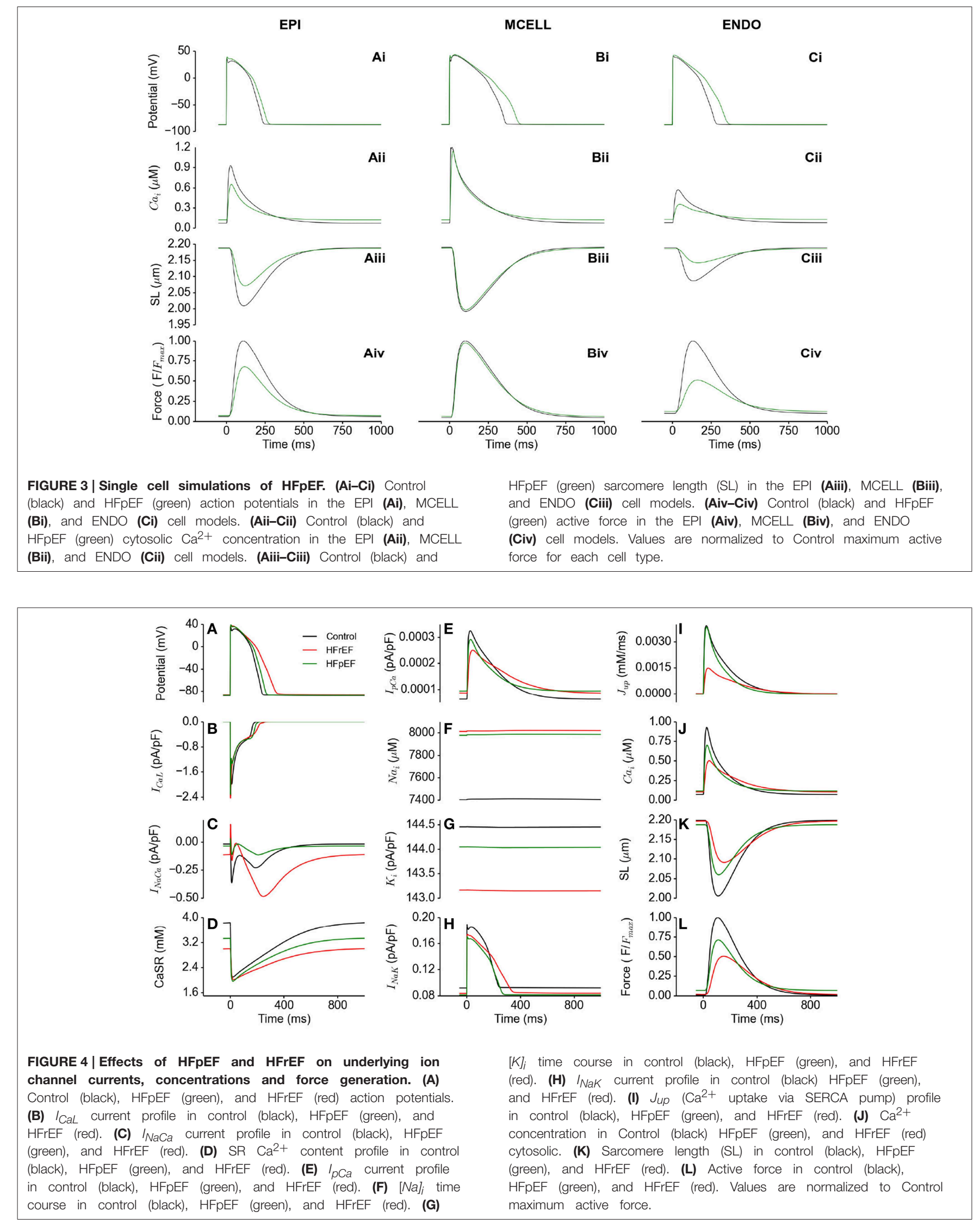

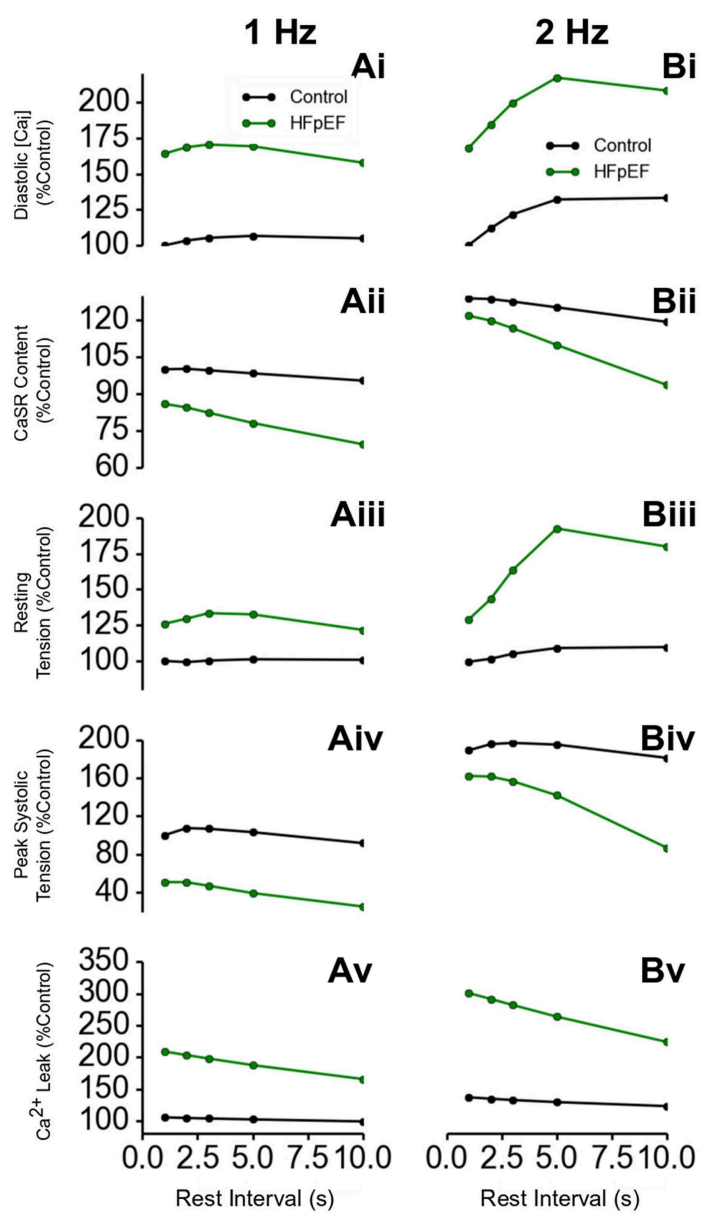

FIGURE 5 | $\mathrm{Ca}^{2+}$ handling and post-rest properties in HFpEF. (Ai,Bi) Diastolic $\mathrm{Ca}^{2+}$ level in HFpEF relative to control at $1 \mathrm{~Hz}(\mathbf{A i})$ and $2 \mathrm{~Hz}(\mathbf{B i})$ pacing rates. (Aii,Bii) $\mathrm{SR} \mathrm{Ca}^{2+}$ content level in $\mathrm{HFpEF}$ relative to control at $1 \mathrm{~Hz}$ (Aii) and $2 \mathrm{~Hz}$ (Bii) pacing rates. (Aiii,Biii) Resting tension in $\mathrm{HFpEF}$ relative to control at $1 \mathrm{~Hz}$ (Aiii) and $2 \mathrm{~Hz}$ (Biii) pacing rates. (Aiv,Biv) Peak systolic tension in $\mathrm{HFpEF}$ relative to control at $1 \mathrm{~Hz}$ (Aiv) and $2 \mathrm{~Hz}$ (Biv) pacing rates. (Av,Bv) SR $\mathrm{Ca}^{2+}$ Ca leak in HFpEF relative to control at $1 \mathrm{~Hz}(\mathbf{A v})$ and $2 \mathrm{~Hz}$ (Bv) pacing rates.

the end-diastolic wall thickness. These simulation results can account for incomplete relaxation and preserved LVEF at the $3 \mathrm{D}$ organ level, whilst at the cellular level, the activation force and cell sarcolemmal shortening are dramatically impaired in the HFpEF condition. This is attributable to the hypertrophied ventricle wall.

\section{Stress Distribution in HFpEF}

Effects of LV wall thickness on the spatial distribution of stress was investigated. Figure 8 shows a 4 -chamber view of the magnitude of the stress distribution across the LV in control and varied hypertrophic conditions (Figures 8A-G). At $0 \mathrm{~ms}$, the stress magnitude across the ventricle was low in all the conditions as excitation had yet to commence (Figure 8A). By $100 \mathrm{~ms}$, when the LV was electrically activated, there was developed stress in all cases with the greatest stress in apex of the MODERATE LV (Figure 8B). At $200 \mathrm{~ms}$ (Figure 8C), there was considerable stress in the LV in all conditions with the greatest stress intensity in SEVERE. The hypertrophic LV also had greater stress intensity in the LV apex and epicardium compared to NORMAL. The situation was similar at $300 \mathrm{~ms}$ (Figure 8D) and $400 \mathrm{~ms}$ (Figure 8E) except that there was progressive relaxation and the stress intensity was less than at $200 \mathrm{~ms}$. By $600 \mathrm{~ms}$ (Figure 8F), stress in most of the NORMAL LV had reduced considerably while the hypertrophic LVs still showed $\sim 25 \%$ stress. At $700 \mathrm{~ms}$, stress in the NORMAL LV was negligible but still about 20\% in the hypertrophic cases. These simulation results showed that increased wall thickness led to increased tissue stress though, at the cellular level, the active force was reduced during the time course of action potentials in HEpEF condition.

\section{Strain Distribution in HFpEF}

Effects of wall thickness on the spatial distribution of strain was also analyzed. Figure 9 also shows a 4-chamber view of the magnitude of the strain distribution across the LV in control and hypertrophic conditions during the time course cardiac excitation (Figures 9A-G). Strain developed from the rest state (Figure 9A) to $\sim 50 \%$ at $100 \mathrm{~ms}$ (Figure 9B) in all cases with the smallest strain magnitude in SEVERE. By $200 \mathrm{~ms}$ (Figure 9C), strain magnitude had reached a distribution of between 50 and $100 \%$ in all cases with SEVERE having the highest strain intensity. This was concentrated in the epicardial to mid-wall regions and the middle segment of the LV. The situation was similar at $300 \mathrm{~ms}$ (Figure 9D) and $400 \mathrm{~ms}$ (Figure 9E) except that the strain intensity was reduced as relaxation was underway. The strain magnitude reduced further at $600 \mathrm{~ms}$ (Figure 9F) to $\sim 10$ $15 \%$ in NORMAL, $\sim 25 \%$ in MILD, $\sim 25-30 \%$ in MODERATE and $\sim 30-35 \%$ in SEVERE. The strain was mainly concentrated in the LV apex in all cases. By $700 \mathrm{~ms}$ (Figure 9G), strain magnitude was $\sim 5 \%$ in NORMAL, $10-25 \%$ in all the hypertrophic cases. These simulation results showed an increased residual of strain in cardiac tissue due to elevated diastolic $\mathrm{Ca}^{2+}$ level, which was consistent with observed incomplete relaxation of the LV in HEpEF condition.

\section{Discussion}

\section{Summary of Major Findings}

In this study we have developed, for the first time, a family of multilevel models for the electro-mechanics of the left ventricle in the setting of HFpEF, at the cellular and 3D organ levels. These models incorporated detailed HFpEF-related ion channel remodeling and impaired $\mathrm{Ca}^{2+}$ homeostasis at the cellular level, and concentric hypertrophy of the left ventricle wall at the organ level. Our major findings are: (i) with impaired $\mathrm{Ca}^{2+}$ handling and ion channel remodeling in HFpEF, the action potential duration of ventricular cells are prolonged, together with an elevated diastolic $\mathrm{Ca}^{2+}$ concentration, but a decreased systolic $\mathrm{Ca}^{2+}$ level. Such an elevated diastolic $\mathrm{Ca}^{2+}$ concentration provides a cellular basis for incomplete ventricular relaxation at the organ level; (ii) at the cellular level the active force and sarcomere length shortening is reduced during the time course of action potentials in HFpEF. However, at the organ level, tissue stress and strain is increased due to the increased wall thickness of 


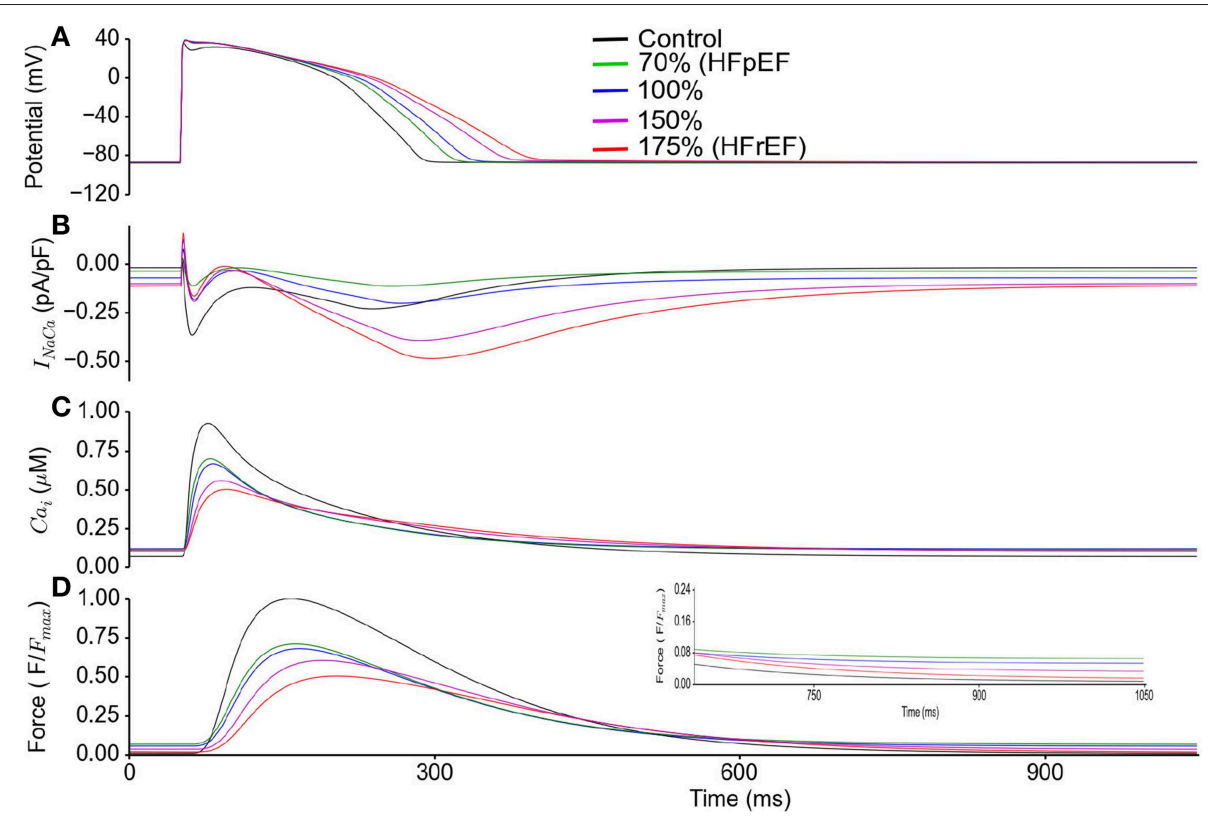

FIGURE 6 | HFpEF Model sensitivity to increasing NCX activity and its influence on incomplete relaxation in the cellular model. Simulation results were compared between control (in absence of ionic current remodeling) and HFpEF condition. HFpEF simulations were performed with parameters as listed in Table 1, but with NCX activity changing from $70 \%$ (HFpEF condition) to 100, 150, and $175 \%$ (HFrEF condition) of the control value. (A) Action potential (B) $I_{\mathrm{NaCa}}$ current profile. (C) $\mathrm{Ca}^{2+}$ concentration. (D) Active force. Values are normalized to maximum active force in control. (Inset: magnified diastolic phase). concentric LV hypertrophy; (iii) the impaired $\mathrm{Ca}^{2+}$ homeostasis becomes more pronounced at high stimulation rates; and (iv) reduction of $I_{\mathrm{NaCa}}$ in the HEpEF model is the most influential factor on impaired relaxation dynamics. Collectively, these simulations predict the key features of HFpEF observed clinically, and also provide insights for understanding the cellular and tissue bases of impaired electro-mechanics of the heart in HFpEF.

\section{Cellular Basis of Impaired Cardiac Electro-Mechanics}

The results of our study suggest that at the cellular level the observed impaired cardiac electro-mechanics (such as reduced cell length shortening and active force) are attributable to alterations in cellular $\mathrm{Ca}^{2+}$ homeostasis and action potentials. In simulations, we observed an elevated diastolic intracellular $\mathrm{Ca}^{2+}$ concentration, but a reduced systolic $\mathrm{Ca}^{2+}$ concentration. The elevated diastolic $\mathrm{Ca}^{2+}$ concentration is due to an increased $\mathrm{Ca}^{2+}$ leak from the SR (see Table 1), and the reduced systolic $\mathrm{Ca}^{2+}$ concentration can be explained by a reduced $\mathrm{Ca}^{2+}$ release from the SR as a consequence of a decreased SR content (Figure 4D). The observed APD prolongation is attributable to the augmented late- $\mathrm{Na}^{+}$current, reduced potassium channel currents and $I_{\mathrm{NaK}}$ as seen in the heart failure condition (see Table 1). These results were more pronounced at a pacing rate of $2 \mathrm{~Hz}$ (Figures $5 \mathbf{B i}-\mathbf{B v}$ ) because of the shorter duration between beats allowing less recovery time for $\mathrm{Ca}^{2+}$ cycling processes. This is related to changes in mechanical and relaxation restitution, which correlate physiologically to the recovery kinetics of $\mathrm{Ca}^{2+}$ release mechanisms and sequestration capacity of the SR (Franz et al., 1983; Burkhoff et al.,
1984; Prabhu and Freeman, 1995; Zaugg et al., 1995; Kjørstad et al., 2007).

In simulations, we also observed an increased intracellular $\mathrm{Na}^{+}$concentration and a reduced intracellular $\mathrm{K}^{+}$concentration (Figures 4F,G), which may be attributable to the augmented $I_{\mathrm{NaL}}$ and reduced $I_{\mathrm{NaK}}$ (see Table $\mathbf{1}$ ). The altered $\mathrm{Na}^{+}$and $\mathrm{K}^{+}$ homeostasis may also impair $\mathrm{Ca}^{2+}$ homeostasis. During the time course of an action potential, there was a reduced $I_{\mathrm{NaK}}$, which also contributed partially to an increase of $\left[\mathrm{Na}^{+}\right]_{i}$ and a decrease in $\left[\mathrm{K}^{+}\right]_{i}$ within the cytosol. NCX extrudes $\mathrm{Ca}^{2+}$ from the cytoplasm and imports $\mathrm{Na}^{+}$into it. However, with the build-up of $\mathrm{Na}^{+}$in the cytoplasm as a result of the reduced activity of the $\mathrm{Na}^{+}-\mathrm{K}^{+}$pump, the forward mode activity of NCX was reduced, but its reverse mode activity was enhanced. This led to an increase in $\mathrm{Na}^{+}$extrusion coupled to $\mathrm{Ca}^{2+}$ import (see Figure 4C) leading to an increased $\mathrm{Ca}^{2+}$ concentration at diastolic phase but a reduced $\mathrm{Ca}^{2+}$ concentration at systolic phase. Consequently, this led to a decreased cell length shortening and active force in the systolic phase.

In addition, our simulation results showed that the SR $\mathrm{Ca}^{2+}$ content was reduced compared to control condition, though the $\mathrm{Ca}^{2+}$ release from the SR was compromised. This is attributable to an excessive $\mathrm{Ca}^{2+}$ leak from the SR. It was shown that SR $\mathrm{Ca}^{2+}$ leak was $\sim 100 \%$ greater in HFpEF as compared to control (Figure 5). The excessive $\mathrm{Ca}^{2+}$ leak from the SR and the reduced activity of NCX produced an increased diastolic $\mathrm{Ca}^{2+}$ level, leading to incomplete relaxation and an increase in the active resting tone. This implies that complete relaxation will never be achieved regardless of the duration of diastole. 


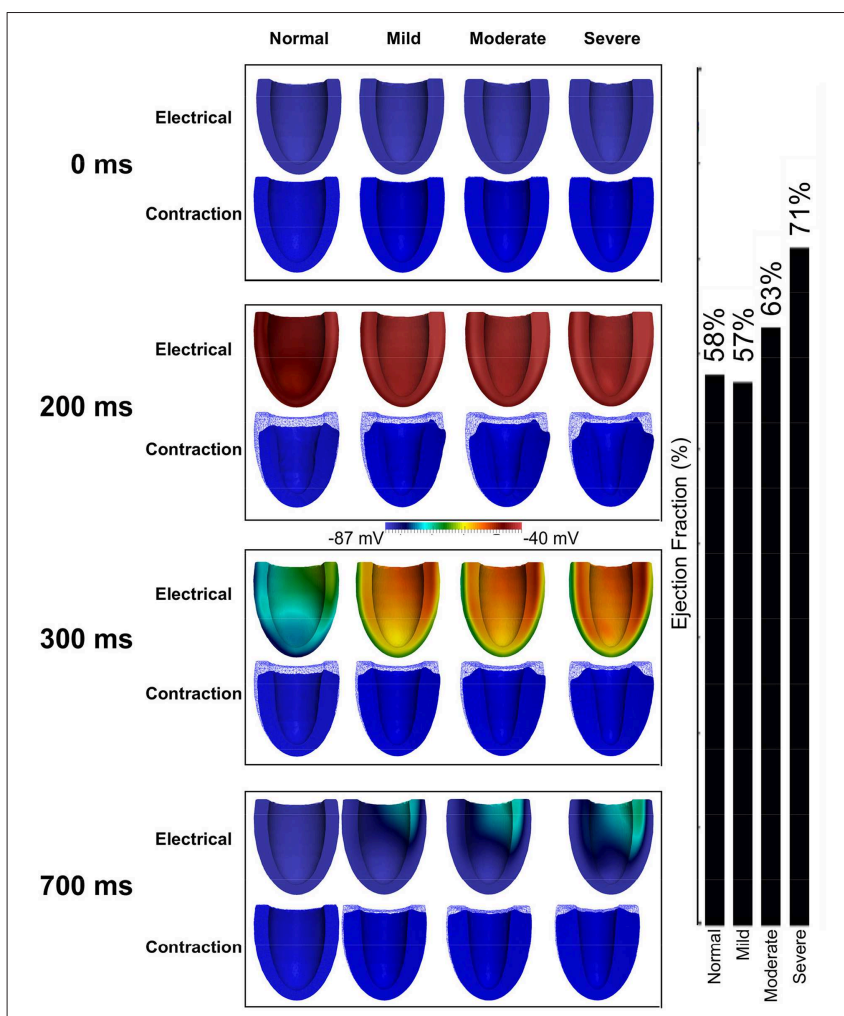

FIGURE 7 | Effects of HFpEF on 3D electro-mechanics. Electrical wave propagation and mechanical contraction at 0,200,300, and $700 \mathrm{~ms}$ in NORMAL, MILD, MODERATE, and SEVERE HFpEF hypertrophic cases. (Far right) Ejection fraction in NORMAL, MILD, MODERATE, and SEVERE HFpEF hypertrophic cases.

The simulation results discussed above were based on our HFpEF model developed from work by Zile and Gaasch (2011) and Selby et al. (2011). To our knowledge these are the only two studies that provide any notion of electrophysiological changes in HFpEF relative to control conditions. As the only difference between the HFpEF and the HFrEF models is a $30 \%$ NCX reduction in the former compared to control and a $175 \%$ increase in the latter (Table 1), we investigated whether changes in NCX activity could convert HFpEF to HFrEF and vice-versa, and therefore affect myocyte relaxation dynamics (Figure 6). This indeed proved to be the case. The results imply that a change in NCX activity is the dominant factor leading to impaired diastolic relaxation in $\mathrm{HFpEF}$.

\section{Effects of Concentric Hypertrophy of the Ventricle Wall on Cardiac Electro-Mechanics}

Increased ventricular wall thickness has dramatic impacts on cardiac electro-mechanics. This is illustrated by the effects of varied degrees of hypertrophy on the ejection fraction in HFpEF condition (Figure 9). In simulations, the wall thickness of the LV varied from $9 \mathrm{~mm}$ (normal) to 12,15 , and $18 \mathrm{~mm}$, mimicking varying degrees of hypertrophy. Though at the cellular level, cell length shortening and active force was decreased in HFpEF condition, the LVEF was preserved and increased with the increase of LV wall thickness. This agrees with what is seen clinically (Borlaug and Paulus, 2010; Phan et al., 2012; Liu et al., 2013; Zouein et al., 2013) and with previous modeling data (MacIver and Townsend, 2008; MacIver, 2010b, 2011). Our simulations confirm that the preserved LVEF in HFpEF is due to the thicker wall of the ventricle arising from concentric hypertrophy.

Taken together, our simulations suggest a possible pathway and mechanism underlying cardiac dysfunction in HFpEF. Any co-morbidities such as diabetes, hypertension, inflammation and/or hypertrophy may cause the ion channel and myofilament remodeling in myocardial cells. Cellular remodeling results in abnormal $\mathrm{Ca}^{2+}$ homeostasis, which in turn lead to abnormalities of contraction and incomplete relaxation of the LV with a persistent active resting tone. These abnormalities combine to increase the length of time to which the myocardium is subjected to stress prolonging systole and resulting in abnormal energy utilization and less efficient ejection of the stroke volume.

\section{Relevance to Previous Studies}

Lacombe et al. (2007) investigated the underlying mechanisms of diastolic dysfunction in type 1 diabetic rats. They observed no significant change in $I_{C a L}$, a reduction in $\mathrm{Ca}^{2+}$ transient amplitude and prolongation in its decay, a reduction in $\mathrm{SR} \mathrm{Ca}^{2+}$ load and a decrease in the expression of sarco(endo)plasmic reticulum $\mathrm{Ca}^{2+}$-ATPase-2a (SERCa) protein levels. They concluded that impairment of $\mathrm{Ca}^{2+}$ reuptake during myocyte relaxation contributed to diastolic dysfunction, with preserved global systolic function.

Selby et al. (2011) carried out a study to evaluate tachycardiainduced relaxation abnormalities in myocardium from patients with a normal ejection fraction. They observed incomplete relaxation with increased diastolic tension development at increasing pacing rates, significant resting tone and disproportionately elevated $\mathrm{Ca}^{2+}$ loads due to reduced sarcolemmal $\mathrm{Ca}^{2+}$ extrusion reserve. However, their patients did not carry a clinical diagnosis of heart failure.

MacIver and Townsend (2008) performed a mathematical study on HFpEF to determine the effect of changes in LV hypertrophy on stroke volume and LVEF. They concluded from their model that the preserved LVEF in HFpEF patients was a result of LV hypertrophy, which amplified absolute radial wall thickening in the setting of reduced long-axis shortening. MacIver also showed, using a simple abstract model, that remodeling was necessary to normalize stroke volume and suggested that regulation of end-diastolic volume was a primary compensatory mechanism in heart failure (MacIver, 2010b). However, their models did not consider the contribution of cardiomyocytes, coupled electrical wave propagation or nonlinear anisotropic cardiac mechanics.

Our simulation results are in agreement with and extend the findings of these previous studies (Lacombe et al., 2007; MacIver and Townsend, 2008; Selby et al., 2011), adding new evidence that impaired $\mathrm{Ca}^{2+}$ homeostasis (such as reduced systolic $\mathrm{Ca}^{2+}$ concentration and elevated diastolic $\mathrm{Ca}^{2+}$ concentration) together with hypertrophied wall underlie the key features of HFpEF with preserved ejection fraction and incomplete end-diastolic relaxation. 


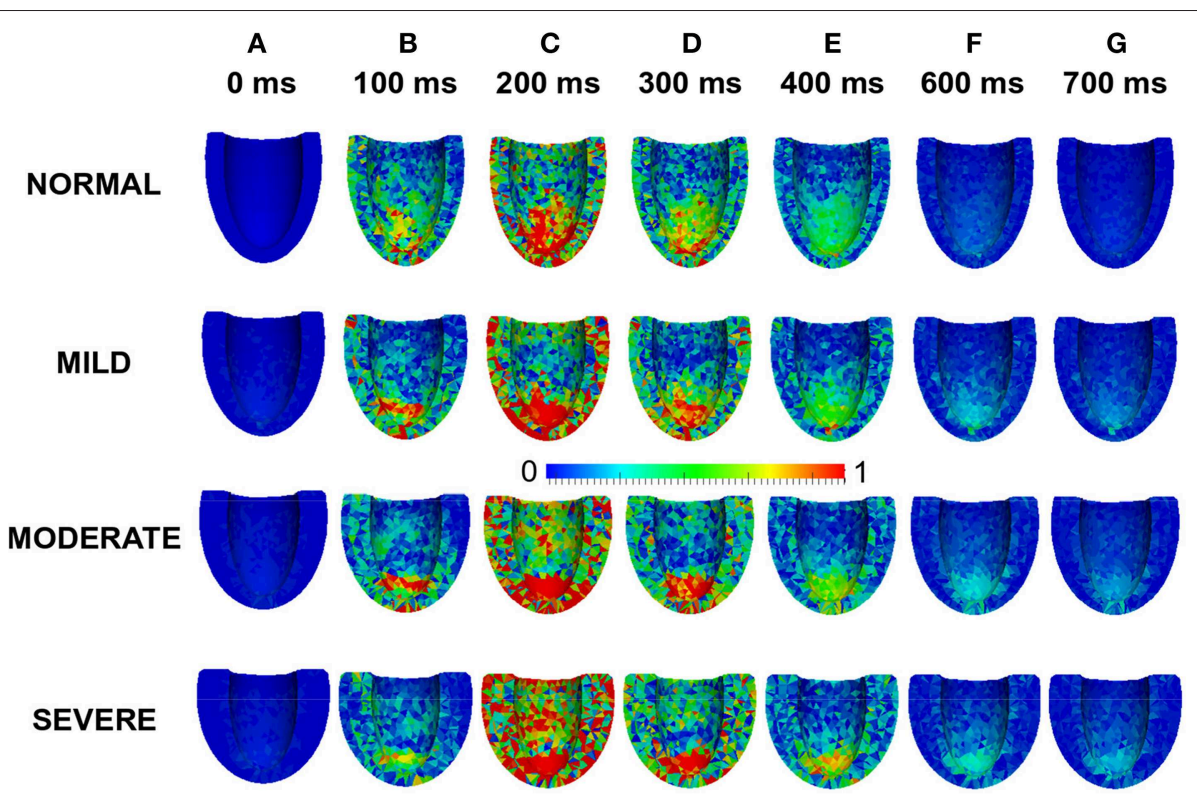

FIGURE 8 | Effects of HFpEF on stress magnitude distribution. Stress magnitude distribution at O ms (A), $100 \mathrm{~ms}$ (B), $200 \mathrm{~ms}$ (C), $300 \mathrm{~ms}$ (D), $400 \mathrm{~ms}$ (E) $600 \mathrm{~ms}$ (F), and $700 \mathrm{~ms}$ (G) in NORMAL, MILD, MODERATE, and SEVERE hypertrophic HFpEF cases.

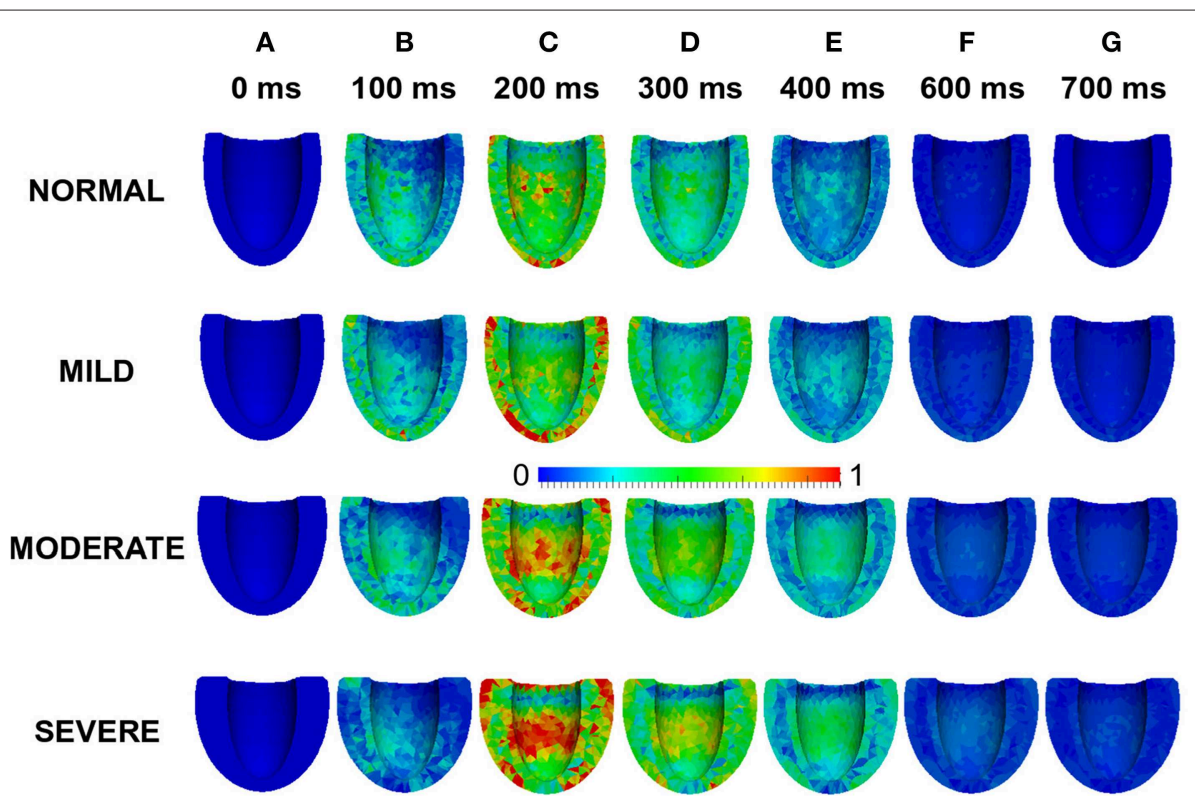

FIGURE 9 | Effects of HFpEF on strain magnitude distribution. Strain magnitude distribution at $0 \mathrm{~ms}$ (A), $100 \mathrm{~ms}$ (B), $200 \mathrm{~ms}$ (C), $300 \mathrm{~ms}$ (D), $400 \mathrm{~ms}$ (E) $600 \mathrm{~ms}$ (F), and $700 \mathrm{~ms}$ (G) in NORMAL, MILD, MODERATE, and SEVERE hypertrophic HFpEF cases.

\section{Limitations}

In addition to acknowledged limitations of both the ORd electrophysiology model (O'Hara et al., 2011) and the (Tran et al., 2010) myofilament model, as experimental data show a reduction in NCX activity in diastolic dysfunction compared to control (Zile and Gaasch, 2011), we made a 30\% reduction in NCX activity in our HFpEF model as there was no quantitative data available at the time of this study. In HFpEF patients, collagen production results in interstitial fibrosis (Heymans et al., 2005; Konstantinou et al., 2013), which we considered by reducing the intracellular electrical conductivities by $20 \%$ due to a lack of quantitative data. The HFpEF model also relaxes somewhat faster than the experimental data, which would imply that the effects of incomplete relaxation would be even greater than we have shown. As there 
are no data available, we assumed the same degree of ion channel remodeling for the hypertrophied ventricles and also assumed the same distribution of ENDO, MCELL, and EPI cell types across the ventricular wall. We also did not consider electromechanical feedback between the electrical wave propagation and mechanically contracting ventricles. Finally, the use of a fluid-structure interaction model with the interaction of blood and the myocardial wall to determine pressure boundary conditions would allow a more realistic pressure profile. Whilst it is important that these potential limitations are stated, they do not fundamentally alter the principal conclusions of this study.

\section{Conclusion}

We have developed a novel, biophysically detailed model of $\mathrm{HFpEF}$ and used it to investigate the cellular mechanisms underlying myocardial $\mathrm{Ca}^{2+}$ homeostasis in HFpEF. We observed an elevated diastolic $\left[\mathrm{Ca}^{2+}\right]_{i}$ level, a reduction in $\mathrm{SR} \mathrm{Ca}^{2+}$ content and reduced $\mathrm{SR} \mathrm{Ca}^{2+}$ release, a reduction in $\mathrm{SR} \mathrm{Ca}^{2+}$

\section{References}

Adeniran, I., Hancox, J. C., and Zhang, H. (2013a). Effect of cardiac ventricular mechanical contraction on the characteristics of the ECG: a simulation study. J. Biomed. Sci. Eng. 6, 47-60. doi: 10.4236/jbise.2013.612A007

Adeniran, I., Hancox, J., and Zhang, H. (2013b). In silico investigation of the short QT syndrome, using human ventricle models incorporating electromechanical coupling. Front. Physiol. 4:166. doi: 10.3389/fphys.2013.00166

Alan, C., and Hindmarsh, P. N. B. (2005). SUNDIALS: suite of nonlinear and differential/algebraic equation solvers. ACM Trans. Math. Softw. 31, 363-396. doi: 10.1145/1089014.1089020

Ambrosi, D., Arioli, G., Nobile, F., and Quarteroni, A. (2011). Electromechanical coupling in cardiac dynamics: the active strain approach. SIAM J. Appl. Math. 71, 605-621. doi: 10.1137/100788379

Asrar Ul Haq, M., Mutha, V., Rudd, N., Hare, D. L., and Wong, C. (2014). Heart failure with preserved ejection fraction-unwinding the diagnosis mystique. Am. J. Cardiovasc. Dis. 4, 100-113.

Auricchio, F., Beirão da Veiga, L., Lovadina, C., and Reali, A. (2010). The importance of the exact satisfaction of the incompressibility constraint in nonlinear elasticity: mixed FEMs versus NURBS-based approximations. Comput. Methods Appl. Mech. Eng. 199, 314-323. doi: 10.1016/j.cma.2008. 06.004

Barry, W. H., and Bridge, J. H. (1993). Intracellular calcium homeostasis in cardiac myocytes. Circulation 87, 1806-1815. doi: 10.1161/01.CIR.87.6.1806

Barry, W. H., Hasin, Y., and Smith, T. W. (1985). Sodium pump inhibition, enhanced calcium influx via sodium-calcium exchange, and positive inotropic response in cultured heart cells. Circ. Res. 56, 231-241. doi: 10.1161/01.RES.56.2.231

Bers, D. (2001). Excitation-Contraction Coupling and Cardiac Contractile Force, 2nd Edn. Dordrecht: Springer.

Bhatia, R. S., Tu, J. V., Lee, D. S., Austin, P. C., Fang, J., Haouzi, A., et al. (2006). Outcome of heart failure with preserved ejection fraction in a population-based study. N. Engl. J. Med. 355, 260-269. doi: 10.1056/NEJMoa051530

Boffi, D., Brezzi, F., and Fortin, M. (2013). Mixed Finite Element Methods and Applications, 2013 Edn. New York, NY: Springer.

Bogaert, J., and Rademakers, F. E. (2001). Regional nonuniformity of normal adult human left ventricle. Am. J. Physiol. Heart Circ. Physiol. 280, H610-H620.

Bonet, J., and Wood, R. D. (2008). Nonlinear Continuum Mechanics for Finite Element Analysis, 2nd Edn. New York, NY: Cambridge University Press.

Borbély, A., van der Velden, J., Papp, Z., Bronzwaer, J. G., Edes, I., Stienen, G. J., et al. (2005). Cardiomyocyte stiffness in diastolic heart failure. Circulation 111, 774-781. doi: 10.1161/01.CIR.0000155257.33485.6D sequestration, an increase in resting tension, incomplete relaxation, reduced systolic stress and prolonged stress and strain durations. These mechanisms suggest that in HFpEF patients, impaired $\mathrm{Ca}^{2+}$ handling principally caused by reduction in NCX activity is a dominant abnormality in the condition that explains the mechanisms for impaired cardiac electro-mechanics in HFpEF.

\section{Acknowledgments}

This work was supported by project grants from Engineering and Physical Science Research Council UK (EP/J00958X/1; $\mathrm{EP} / \mathrm{I029826/1).}$

\section{Supplementary Material}

The Supplementary Material for this article can be found online at: http://www.frontiersin.org/journal/10.3389/fphys. 2015.00078/abstract

Borlaug, B. A., and Paulus, W. J. (2010). Heart failure with preserved ejection fraction: pathophysiology, diagnosis, and treatment. Eur. Heart J. 32, 670-679. doi: 10.1093/eurheartj/ehq426

Braess, D. (2007). Finite Elements: Theory, Fast Solvers, and Applications in Solid Mechanics, $3 r d E d n$. New York, NY: Cambridge University Press.

Braess, D., and Ming, P. (2005). A finite element method for nearly incompressible elasticity problems. Math. Comput. 74, 25-52. doi: 10.1090/S0025-571804-01662-X

Brenner, S. C., and Scott, R. (2010). The Mathematical Theory of Finite Element Methods, 3rd Edn. 2008. New York, NY: Springer.

Bueno-Orovio, A., Sánchez, C., Pueyo, E., and Rodriguez, B. (2014). Na/K pump regulation of cardiac repolarization: insights from a systems biology approach. Pflügers. Arch. Eur. J. Physiol. 466, 183-193. doi: 10.1007/s00424-013-1293-1

Burkhoff, D., Yue, D. T., Franz, M. R., Hunter, W. C., and Sagawa, K. (1984). Mechanical restitution of isolated perfused canine left ventricles. Am. J. Physiol. 246, H8-H16.

Burnett, D. S. (1987). Finite Element Analysis: From Concepts to Applications, 1st Edn. Reading, MA: Addison Wesley.

Chamberland, E., Fortin, A., and Fortin, M. (2010). Comparison of the performance of some finite element discretizations for large deformation elasticity problems. Comput. Struct. 88, 664-673. doi: 10.1016/j.compstruc.2010.02.007

Cherubini, C., Filippi, S., Nardinocchi, P., and Teresi, L. (2008). An electromechanical model of cardiac tissue: constitutive issues and electrophysiological effects. Prog. Biophys. Mol. Biol. 97, 562-573. doi: 10.1016/j.pbiomolbio.2008.02.001

Ciarlet, P. G. (2002). The Finite Element Method for Elliptic Problems, 2nd Edn. New York, NY: Society for Industrial and Applied Mathematics.

Cohen, S., and Hindmarsh, A. C. (1996). "Cvode, a stiff/nonstiff ode solver in C," in C. Computers in Physics, ed L. M. Holmes (New York, NY: American Institute of Physics Inc.), 138-143.

Colli Franzone, P., Pavarino, L. F., and Taccardi, B. (2005). Simulating patterns of excitation, repolarization and action potential duration with cardiac Bidomain and Monodomain models. Math. Biosci. 197, 35-66. doi: 10.1016/j.mbs.2005.04.003

Costa, K. D., Holmes, J. W., and McCulloch, A. D. (2001). Modelling cardiac mechanical properties in three dimensions. Philos. Trans. R. Soc. Lond. Ser. Math. Phys. Eng. Sci. 359, 1233-1250. doi: 10.1098/rsta.2001.0828

Drouin, E., Charpentier, F., Gauthier, C., Laurent, K., and Le Marec, H. (1995). Electrophysiologic characteristics of cells spanning the left ventricular wall of human heart: evidence for presence of M cells. J. Am. Coll. Cardiol. 26, 185-192. doi: 10.1016/0735-1097(95)00167-X

Ebashi, S. (1984). $\mathrm{Ca}^{2+}$ and the contractile proteins. J. Mol. Cell. Cardiol. 16, 129-136. doi: 10.1016/S0022-2828(84)80701-4 
Ern, A., and Guermond, J.-L. (2010). Theory and Practice of Finite Elements, 1st Edn. 2004. New York, NY: Springer.

Franz, M. R., Schaefer, J., Schöttler, M., Seed, W. A., and Noble, M. I. (1983). Electrical and mechanical restitution of the human heart at different rates of stimulation. Circ. Res. 53, 815-822. doi: 10.1161/01.RES.53.6.815

Glitsch, H. G. (2001). Electrophysiology of the sodium-potassium-ATPase in cardiac cells. Physiol. Rev. 81, 1791-1826.

Gomez, J. F., Cardona, K., Romero, L., Ferrero, J. M., and Trenor, B. (2014). Electrophysiological and structural remodeling in heart failure modulate arrhythmogenesis. 1D simulation study. PLoS ONE 9:e106602. doi: 10.1371/journal.pone. 0106602

Haga, J. B., Osnes, H., and Langtangen, H. P. (2012). On the causes of pressure oscillations in low-permeable and low-compressible porous media. Int. J. Numer. Anal. Methods Geomech. 36, 1507-1522. doi: 10.1002/ nag. 1062

Heymans, S., Schroen, B., Vermeersch, P., Milting, H., Gao, F., Kassner, A., et al. (2005). Increased cardiac expression of tissue inhibitor of metalloproteinase1 and tissue inhibitor of metalloproteinase- 2 is related to cardiac fibrosis and dysfunction in the chronic pressure-overloaded human heart. Circulation 112, 1136-1144. doi: 10.1161/CIRCULATIONAHA.104.516963

Holzapfel, G. A. (2000). Nonlinear Solid Mechanics: A Continuum Approach for Engineering, 1st Edn. Chichester: Wiley.

Hughes, T. J. R. (2000). The Finite Element Method: Linear Static and Dynamic Finite Element Analysis. New York, NY: Dover Publications.

Keener, J., and Sneyd, J. (2008). Mathematical Physiology: II: Systems Physiology, 2nd Edn. New York, NY: Springer.

Keldermann, R. H., Nash, M. P., and Panfilov, A. V. (2009). Modeling cardiac mechano-electrical feedback using reaction-diffusion-mechanics systems. Phys. Nonlinear Phenom. 238, 1000-1007. doi: 10.1016/j.physd.2008.08.017

Keller, D. U. J., Kalayciyan, R., Dössel, O., and Seemann, G. (2009). "Fast creation of endocardial stimulation profiles for the realistic simulation of body surface ECGs," in IFMBE Proceedings, 145-148. Available online at: http://www.scopus.com/inward/record.url?eid=2-s2.0-77950134835\&partner ID $=40 \& m d 5=2 \mathrm{db} 2 \mathrm{~b} 89 \mathrm{cc} 19 \mathrm{de} 5 \mathrm{e} 28 \mathrm{ec} 01$ ebbf0dffff0 (Accessed July 20, 2011).

Kjørstad, K. E., Nordhaug, D. O., Korvald, C., Müller, S., Steensrud, T., and Myrmel, T. (2007). Mechanical restitution curves: a possible load independent assessment of contractile function. Eur. J. Cardio-Thorac. Surg. 31, 677-684. doi: 10.1016/j.ejcts.2007.01.013

Kono, M., Kisanuki, A., Takasaki, K., Nakashiki, K., Yuasa, T., Kuwahara, E., et al. (2009). Left ventricular systolic function is abnormal in diastolic heart failure: re-assessment of systolic function using cardiac time interval analysis. J. Cardiol. 53, 437-446. doi: 10.1016/j.jjcc.2009.02.014

Konstantinou, D. M., Chatzizisis, Y. S., and Giannoglou, G. D. (2013). Pathophysiology-based novel pharmacotherapy for heart failure with preserved ejection fraction. Pharmacol. Ther. 140, 156-166. doi: 10.1016/j.pharmthera.2013.05.012

Lacombe, V. A., Viatchenko-Karpinski, S., Terentyev, D., Sridhar, A., Emani, S., Bonagura, J. D., et al. (2007). Mechanisms of impaired calcium handling underlying subclinical diastolic dysfunction in diabetes. Am. J. Physiol. Regul. Integr. Comp. Physiol. 293, R1787-R1797. doi: 10.1152/ajpregu.00059.2007

Le Tallec, P. (1994). "Numerical methods for nonlinear three-dimensional elasticity," in Handbook of Numerical Analysis, Vol. III, eds P. G. Ciarlet and J. L. Lions (North-Holland: Amsterdam), 465-622.

Li, G. R., Feng, J., Yue, L., and Carrier, M. (1998). Transmural heterogeneity of action potentials and Itol in myocytes isolated from the human right ventricle. Am. J. Physiol. 275, H369-H377.

Liu, Y., Haddad, T., and Dwivedi, G. (2013). Heart failure with preserved ejection fraction: current understanding and emerging concepts. Curr. Opin. Cardiol. 28, 187-196. doi: 10.1097/HCO.0b013e32835c5492

Logg, A., Mardal, K.-A., and Wells, G. (eds.) (2012). Automated Solution of Differential Equations by the Finite Element Method: The FEniCS Book, 2012th Edn. Heidelberg: Springer.

MacIver, D. H. (2010a). Current controversies in heart failure with a preserved ejection fraction. Future Cardiol. 6, 97-111. doi: 10.2217/fca.09.56

MacIver, D. H. (2010b). Is remodeling the dominant compensatory mechanism in both chronic heart failure with preserved and reduced left ventricular ejection fraction? Basic Res. Cardiol. 105, 227-234. doi: 10.1007/s00395-0090063-x
MacIver, D. H. (2011). A new method for quantification of left ventricular systolic function using a corrected ejection fraction. Eur. J. Echocardiogr. 12, 228-234. doi: 10.1093/ejechocard/jeq185

MacIver, D. H., and Dayer, M. J. (2012). An alternative approach to understanding the pathophysiological mechanisms of chronic heart failure. Int. J. Cardiol. 154, 102-110. doi: 10.1016/j.ijcard.2011.05.075

MacIver, D. H., and Townsend, M. (2008). A novel mechanism of heart failure with normal ejection fraction. Heart Br. Card. Soc. 94, 446-449. doi: $10.1136 /$ hrt.2006.114082

Marsden, J. E., and Hughes, T. J. R. (1994). Mathematical Foundations of Elasticity. New Jersey: Dover Publications.

Niederer, S. A., and Smith, N. P. (2008). An improved numerical method for strong coupling of excitation and contraction models in the heart. Prog. Biophys. Mol. Biol. 96, 90-111. doi: 10.1016/j.pbiomolbio.2007.08.001

O’Hara, T., Virág, L., Varró, A., and Rudy, Y. (2011). Simulation of the undiseased human cardiac ventricular action potential: model formulation and experimental validation. PLoS Comput. Biol. 7:e1002061. doi: 10.1371/journal.pcbi. 1002061

Owan, T. E., Hodge, D. O., Herges, R. M., Jacobsen, S. J., Roger, V. L., and Redfield, M. M. (2006). Trends in prevalence and outcome of heart failure with preserved ejection fraction. N. Engl. J. Med. 355, 251-259. doi: 10.1056/NEJMoa052256

Pathmanathan, P., and Whiteley, J. P. (2009). A numerical method for cardiac mechanoelectric simulations. Ann. Biomed. Eng. 37, 860-873. doi: 10.1007/s10439-009-9663-8

Phan, T. T., Shivu, G. N., Abozguia, K., Sanderson, J. E., and Frenneaux, M. (2012). The pathophysiology of heart failure with preserved ejection fraction: from molecular mechanisms to exercise haemodynamics. Int. J. Cardiol. 158, 337-343. doi: 10.1016/j.ijcard.2011.06.113

Prabhu, S. D., and Freeman, G. L. (1995). Effect of tachycardia heart failure on the restitution of left ventricular function in closed-chest dogs. Circulation 91, 176-185. doi: 10.1161/01.CIR.91.1.176

Rademakers, F. E., Rogers, W. J., Guier, W. H., Hutchins, G. M., Siu, C. O., Weisfeldt, M. L., et al. (1994). Relation of regional cross-fiber shortening to wall thickening in the intact heart. Three-dimensional strain analysis by NMR tagging. Circulation 89, 1174-1182. doi: 10.1161/01.CIR.89.3.1174

Redfield, M. M., Jacobsen, S. J., Burnett, J. C., Mahoney, D. W., Bailey, K. R., and Rodeheffer, R. J. (2003). Burden of systolic and diastolic ventricular dysfunction in the community: appreciating the scope of the heart failure epidemic. JAMA 289, 194-202. doi: 10.1001/jama.289.2.194

Rice, J. J., Wang, F., Bers, D. M., and de Tombe, P. P. (2008). Approximate model of cooperative activation and crossbridge cycling in cardiac muscle using ordinary differential equations. Biophys. J. 95, 2368-2390. doi: 10.1529/biophysj.107.119487

Robertson, S. P., Johnson, J. D., Holroyde, M. J., Kranias, E. G., Potter, J. D., and Solaro, R. J. (1982). The effect of troponin I phosphorylation on the $\mathrm{Ca}^{2+}$ binding properties of the $\mathrm{Ca}^{2+}$-regulatory site of bovine cardiac troponin. J. Biol. Chem. 257, 260-263.

Rossi, S., Lassila, T., Ruiz-Baier, R., Sequeira, A., and Quarteroni, A. (2014). Thermodynamically consistent orthotropic activation model capturing ventricular systolic wall thickening in cardiac electromechanics. Eur. J. Mech. ASolids. 48, 129-142. doi: 10.1016/j.euromechsol.2013.10.009

Rush, S., and Larsen, H. (1978). A practical algorithm for solving dynamic membrane equations. IEEE Trans. Biomed. Eng. 25, 389-392. doi: 10.1109/TBME.1978.326270

Sanderson, J. E. (2007). Heart failure with a normal ejection fraction. Heart 93, 155-158. doi: 10.1136/hrt.2005.074187

Selby, D. E., Palmer, B. M., LeWinter, M. M., and Meyer, M. (2011). Tachycardiainduced diastolic dysfunction and resting tone in myocardium from patients with a normal ejection fraction. J. Am. Coll. Cardiol. 58, 147-154. doi: 10.1016/j.jacc.2010.10.069

Soma, J. (2011). Heart failure with preserved left ventricular ejection fraction: concepts, misconceptions and future directions. Blood Press. 20, 129-133. doi: $10.3109 / 08037051.2010 .542642$

Sundnes, J., Lines, G. T., and Tveito, A. (2005). An operator splitting method for solving the bidomain equations coupled to a volume conductor model for the torso. Math. Biosci. 194, 233-248. doi: 10.1016/j.mbs.2005.01.001

Taggart, P., Sutton, P. M., Opthof, T., Coronel, R., Trimlett, R., Pugsley, W., et al. (2000). Inhomogeneous transmural conduction during early ischaemia 
in patients with coronary artery disease. J. Mol. Cell. Cardiol. 32, 621-630. doi: 10.1006/jmcc.2000.1105

Tran, K., Smith, N. P., Loiselle, D. S., and Crampin, E. J. (2010). A metabolitesensitive, thermodynamically constrained model of cardiac cross-bridge cycling: implications for force development during ischemia. Biophys. J. 98, 267-276. doi: 10.1016/j.bpj.2009.10.011

Trenor, B., Cardona, K., Gomez, J. F., Rajamani, S., Ferrero, J. M., Belardinelli, L., et al. (2012). Simulation and mechanistic investigation of the arrhythmogenic role of the late sodium current in human heart failure. PLOS ONE 7:e32659. doi: 10.1371/journal.pone.0032659

Vasan, R. S., and Levy, D. (2000). Defining diastolic heart failure: a call for standardized diagnostic criteria. Circulation 101, 2118-2121. doi: 10.1161/01.CIR.101.17.2118

Wang, J., Khoury, D. S., Yue, Y., Torre-Amione, G., and Nagueh, S. F. (2008), Preserved left ventricular twist and circumferential deformation, but depressed longitudinal and radial deformation in patients with diastolic heart failure. Eur. Heart J. 29, 1283-1289. doi: 10.1093/eurheartj/ehn141

Whiteley, J. P., Bishop, M. J., and Gavaghan, D. J. (2007). Soft tissue modelling of cardiac fibres for use in coupled mechano-electric simulations. Bull. Math. Biol. 69, 2199-2225. doi: 10.1007/s11538-007-9213-1

Wilson, L. D., Jennings, M. M., and Rosenbaum, D. S. (2011). Point: M cells are present in the ventricular myocardium. Heart Rhythm 8, 930-933. doi: 10.1016/j.hrthm.2011.01.026

Workman, A. J., Kane, K. A., and Rankin, A. C. (2003). Characterisation of the $\mathrm{Na}, \mathrm{K}$ pump current in atrial cells from patients with and without chronic atrial fibrillation. Cardiovasc. Res. 59, 593-602. doi: 10.1016/S0008-6363(03) 00466-8

Yturralde, R. F., and Gaasch, W. H. (2005). Diagnostic criteria for diastolic heart failure. Prog. Cardiovasc. Dis. 47, 314-319. doi: 10.1016/j.pcad.2005.02.007
Zaugg, C. E., Kojima, S., Wu, S. T., Wikman Coffelt, J., Parmley, W. W., and Buser, P. T. (1995). 997-86 intracellular calcium transients underlie mechanical restitution in whole rat hearts. J. Am. Coll. Cardiol. 25, 325A. doi: 10.1016/07351097(95)92811-I

Zile, M. R., Baicu, C. F., and Gaasch, W. H. (2004). Diastolic heart failureabnormalities in active relaxation and passive stiffness of the left ventricle. N. Engl. J. Med. 350, 1953-1959. doi: 10.1056/NEJMoa032566

Zile, M. R., and Gaasch, W. H. (2011). Abnormal calcium homeostasis: one mechanism in diastolic heart failure. J. Am. Coll. Cardiol. 58, 155-157. doi: 10.1016/j.jacc.2010.10.068

Zile, M. R., Gaasch, W. H., Carroll, J. D., Feldman, M. D., Aurigemma, G. P., Schaer, G. L., et al. (2001). Heart failure with a normal ejection fraction: is measurement of diastolic function necessary to make the diagnosis of diastolic heart failure? Circulation 104, 779-782. doi: 10.1161/hc3201.094226

Zouein, F. A., de Castro Brás, L. E., da Costa, D. V., Lindsey, M. L., Kurdi, M., and Booz, G. W. (2013). Heart failure with preserved ejection fraction: emerging drug strategies. J. Cardiovasc. Pharmacol. 62, 13-21. doi: 10.1097/FJC.0b013e31829a4e61

Conflict of Interest Statement: The authors declare that the research was conducted in the absence of any commercial or financial relationships that could be construed as a potential conflict of interest.

Copyright (c) 2015 Adeniran, MacIver, Hancox and Zhang. This is an open-access article distributed under the terms of the Creative Commons Attribution License (CC $B Y)$. The use, distribution or reproduction in other forums is permitted, provided the original author(s) or licensor are credited and that the original publication in this journal is cited, in accordance with accepted academic practice. No use, distribution or reproduction is permitted which does not comply with these terms. 